\title{
Association Between Melanoma Risk and Height: A Narrative Review
}

\author{
Gino A. Vena ${ }^{1,2}$, Nicoletta Cassano ${ }^{1,2}$, Stefano Caccavale ${ }^{3}$, Giuseppe Argenziano ${ }^{3}$
}

1 Dermatology and Venereology Private Practice, Bari, Italy

2 Dermatology and Venereology Private Practice, Barletta, Italy

3 Dermatology Unit, University of Campania Luigi Vanvitelli, Naples, Italy

Key words: cutaneous melanoma, height, cancer, risk, association

Citation: Vena GA, Cassano N, Caccavale S, Argenziano G. Association between melanoma risk and height: a narrative review. Dermatol Pract Concept. 2019;9(2):82-89. DOI: https://doi.org/10.5826/dpc.0902a02

Accepted: February 22, 2019; Published: April 30, 2019

Copyright: $\odot 2019$ Vena et al. This is an open-access article distributed under the terms of the Creative Commons Attribution License, which permits unrestricted use, distribution, and reproduction in any medium, provided the original author and source are credited.

Funding: None.

Competing interests: The authors have no conflicts of interest to disclose.

Authorship: All authors have contributed significantly to this publication.

Corresponding author: Stefano Caccavale, MD, Dermatology Unit, Department of Mental and Physical Health and Preventive Medicine, University of Campania Luigi Vanvitelli, Via Sergio Pansini, 5, 80131 Naples, Italy. Email: stefano85med@gmail.com

ABSTRACT The link between anthropometric indices, including height, and cancer risk and/or progression has attracted considerable interest in recent years. Adult height results from the complex interplay between genetic, hormonal, nutritional, and other environmental factors and has been found to contribute to the risk of several selected malignancies, although it has not been implicated as a real cause per se. A number of studies have investigated the height-melanoma relationship, showing controversial results so far. In this review, we summarize the epidemiological data regarding the association between height and melanoma risk and analyze the potential underlying mechanisms.

\section{Introduction}

In recent years there has been growing interest in investigating the link between anthropometric measures and cancer risk and progression. Height is included among these measures and has been suggested to contribute to the risk of several selected malignancies, although it has not been implicated as a real cause per se. Adult attained height might indeed be an indirect marker resulting from shared mechanisms within the complex interplay between genetic, hormonal, nutritional, and other environmental factors [1]. Epidemiological studies have also connected the risk of skin cancer to multiple anthropometric parameters, including height, but the results appear to be still controversial and the possible mechanisms underlying the positive association remain unclear.

Cutaneous melanoma is a potentially lethal malignancy derived from melanocytes, whose incidence has steadily increased worldwide over recent decades. The main risk factors include exposure to ultraviolet rays (UVR), number of common and atypical nevi, UVR sensitivity-related phenotypic characteristics, genetic susceptibility, and a family history of melanoma [2].

We conducted a narrative review to summarize the epidemiological data regarding the association between melanoma risk and height. Underlying mechanisms that might explain this association are also discussed. 


\section{Methods}

Literature searches were performed in PubMed and Google Scholar databases up to September 30, 2018, using the terms "skin cancer," "melanoma," and "height." Case-control and cohort studies that investigated the association between cutaneous melanoma risk and height were considered and selected after reading the title and abstract and, when appropriate, the full text. Only articles written in English were chosen. Additional studies were found among the references of the retrieved articles.

Results are described in separate sections depending on whether they were obtained from studies evaluating the risk of cancer at multiple sites, also including melanoma, or analyzing the risk of melanoma only.

\section{Studies Assessing Cancer at Multiple Sites, Including Melanoma}

Several studies have examined the cancer-height relationship and disclosed a significant positive association of adult height with both the overall cancer risk and the incidence of cancer at various anatomical sites, also highlighting a significant association between height and melanoma in both men and women [3-6].

Results from a prospective cohort study in subjects living in the area of Washington State indicated a hazard ratio for melanoma per 5 inches of height of 1.28 with a $95 \%$ confidence interval (CI) of 1.05-1.55, after adjustment for age, sex, and race [3]. In the Institutes of Health-AARP Diet and Health Study, the hazard ratio per $10-\mathrm{cm}$ increase of height was 1.18 (95\% CI 1.13-1.23) in males and 1.14 (95\% CI 1.05-1.24) in females, after adjustment for covariates (age at entry, education, race, smoking status, and body mass index) [5].

Other authors corroborated the existence of this association in particular groups, such as middle-aged women and postmenopausal women [7-10].

The relation between height and cumulative relative risk of cancer appeared to be similar in different populations, with little difference across North America, Europe, Australasia, and Asia [7]. When height was handled as a time-dependent covariate, the results for all cancers combined and melanoma did not differ from those obtained using only baseline height [8].

Melanoma was among the highest ranking sites across the studies that included women and also among men, even after controlling for potential confounding variables, such as body mass index $[4,7,9]$. In a large prospective study involving 7 cohorts in Austria, Norway, and Sweden [4], increased height (per 5-cm increment) was associated with a higher global cancer risk and, among the different cancer types, melanoma had the highest hazard ratio in both women $(1.17,95 \%$ CI 1.11-1.24) and men (1.12, $95 \%$ CI 1.08-1.19).

Height was considered an important explanatory factor accounting for $33.8 \%$ of the excess risk of incident cancer for men vs women overall and for specific anatomical sites, and this was particularly evident also for melanoma, where the proportion mediated by height was $57.3 \%$ [3].

\section{Influence on Mortality}

Adult height appears to have opposite relationships with risk of death from various major causes of chronic diseases and cancer [11].

In the pooled cohort study carried out by Wirén et al [4], height was associated with a nonsignificant increase in the risk of death from cutaneous melanoma in both men and women (hazard ratio per $5-\mathrm{cm}$ increment in height of 1.10 [95\% CI 0.99-1.21] and 1.09 [95\% CI 0.92-1.29], respectively).

An analysis of multiple prospective cohorts involving more than 1 million adults, mostly from Europe and North America, showed a hazard ratio per $6.5-\mathrm{cm}$ greater height of 1.26 (95\% CI 1.12-1.42) for risk of melanoma death, corresponding to the highest relative risk of cancer death in women and men combined [11].

In a prospective study, more than 18,000 London-based male government employees were followed up for mortality for a maximum of 35 years [12]. Following adjustment for covariates, taller men experienced elevated mortality rates from cancer of combined sites and from certain organ-specific malignancies. There was a significant association between height and mortality rate from skin cancer, defined as "malignant melanoma of skin" combined with "other malignant neoplasms of skin" (hazard ratio per 5 -cm increase in height $1.35,95 \%$ CI 1.06-1.70).

\section{Studies Specifically Focusing on Melanoma}

Various case-control and cohort studies analyzed the relation between anthropometric factors, including height, and melanoma risk selectively. Table 1 reports the general characteristics of the studies that focused on the risk of melanoma in association with adult height [13-25]. All these studies analyzed the risk of melanoma only, with the exception of one [24] that evaluated the risk of keratinocyte cancers and melanoma.

Table 2 summarizes the results concerning the association between melanoma risk and height. Despite their methodological heterogeneity, many reports suggested an increased risk of melanoma with increasing height [13-15,18,21-23,25]. 


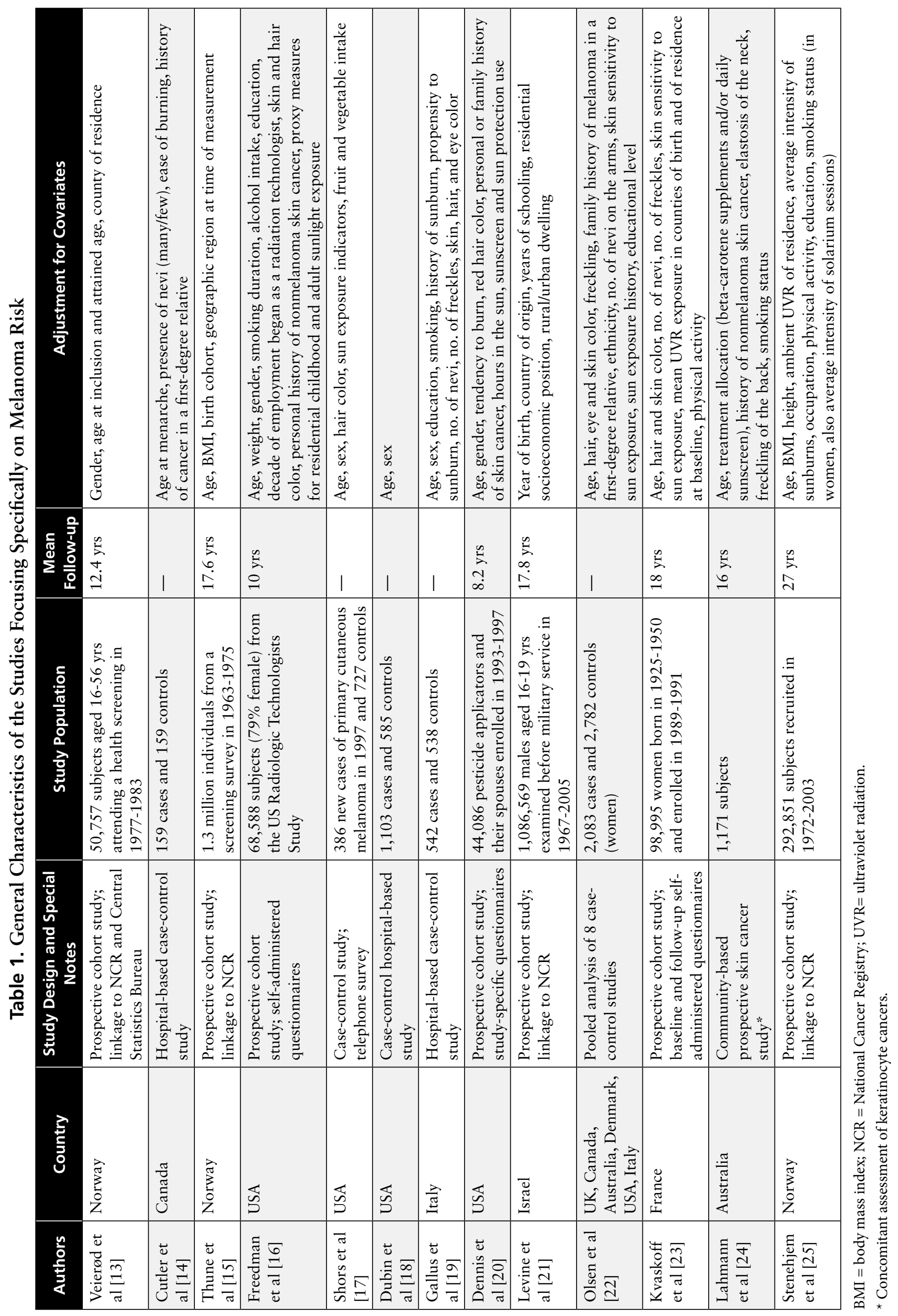


Table 2. Studies Focusing Specifically on Melanoma Risk: Summary of Results Regarding Association With Height

\begin{tabular}{|c|c|}
\hline Study & Main Results \\
\hline Veierød et al [13] & Increased risk with increasing height $(\mathrm{P}<0.01$, test for linear trend $)$ \\
\hline Cutler et al [14] & $\begin{array}{l}\text { Height as a significant risk factor (difference of mean heights in cases vs } \\
\text { controls of only } 2 \mathrm{~cm}, \mathrm{P}=0.009 \text { ) }\end{array}$ \\
\hline Thune et al [15] & $\begin{array}{l}\text { Significant increase of risk with increasing height in both sexes (RR 1.6, } \\
95 \% \text { CI 1.4-1.8 in the highest quintile) }\end{array}$ \\
\hline Freedman et al [16] & No association in men and women \\
\hline Shors et al [17] & $\begin{array}{l}\text { Increased risk for men in the highest vs the lowest quartile (OR 2.4, } \\
95 \% \text { CI 1.3-4.5); no association in women }\end{array}$ \\
\hline Dubin et al [18] & $\begin{array}{l}\text { Increasing linear trend in risk for increasing height }(P<0.005) \text {; RR } 1.62 \\
(95 \% \text { CI 1.01-2.6) in the highest quintile }\end{array}$ \\
\hline Gallus et al [19] & No significant association \\
\hline Dennis et al [20] & No significant association (results not further specified) \\
\hline Levine et al [21] & $\begin{array}{l}\text { Increasing trend in risk for increasing height; HR in the highest quintile } \\
2.42(95 \% \text { CI } 2.06-2.83) \text { in univariate analysis, } 1.40 \text { (95\% CI } 1.19- \\
1.65) \text { in multivariable analysis }\end{array}$ \\
\hline Olsen et al [22] & Increased risk in the highest quartile (pooled OR $1.3,95 \%$ CI $1.1-1.6$ ) \\
\hline Kvaskoff et al [23] & $\begin{array}{l}\text { Positive association in age-adjusted models only (RR } 1.27,95 \% \text { CI } \\
1.05-1.55 \text { for } \geq 164 \mathrm{~cm} \text { vs }<160 \mathrm{~cm} \text {, P for trend }=0.02)\end{array}$ \\
\hline Lahmann et al [24] & $\begin{array}{l}\text { Positive association in men (RR per } 5 \text {-cm increment } 1.55,95 \% \text { CI } 0.97 \text { - } \\
2.47 \text { ), though not significant }\end{array}$ \\
\hline Stenehjem et al [25] & $\begin{array}{l}\text { Positive association in both men and women }\left(\mathrm{P}_{\text {trend }}<0.001\right) ; \mathrm{HR} \text { in the } \\
\text { highest quintile } 1.55(95 \% \text { CI } 1.36-1.77) \text { for men and } 1.52(95 \% \text { CI } \\
\text { 1.31-1.76) for women in multivariable analysis }\end{array}$ \\
\hline
\end{tabular}

$\mathrm{CI}=$ confidence interval $\mathrm{HR}=$ hazard ratio $\mathrm{OR}=$ odds ratio $\mathrm{RR}=$ relative risk .

On the contrary, other studies failed to disclose a significant association [16,19,20,24]. Shors et al observed no association between melanoma and height in women but found an increased risk for men in the highest vs the lowest quartile (odds ratio 2.4, 95\% CI 1.3-4.5), although these results were attenuated after adjustment for weight (odds ratio 1.9, 95\% CI 0.9-3.8) [17]. It is interesting that prospective studies involving large cohorts linked to national cancer registries and followed up for many years consistently reported a significant trend in melanoma-height association [13,15,21,25].

Stenehjem et al recently published the results of a prospective study aimed at assessing the risk of melanoma according to anthropometric factors, adjusted for exposure to UVR, in a large population-based cohort in Norway, linked to the national cancer registry, over an average follow-up period of 27 years [25]. They found significant positive associations for height, with greater than $50 \%$ increased melanoma risk for the highest quintile compared with the lowest quintile in both sexes. Height above the median was estimated to account for $10 \%$ of the male and $6 \%$ of the female melanoma cases, respectively. The authors also used restricted cubic splines for the assessment of melanoma risk. The shape of the risk curve appeared to support an exponential increase. The risk according to height increased with statistical significance until $195 \mathrm{~cm}$ in men and from approximately 170 to $180 \mathrm{~cm}$ in women, and then declined.

Results from a cohort of French women indicated that melanoma risk was positively associated with height in age-adjusted models only, as well as with sitting-to-standing height ratio in multivariable models, while no significant association with sitting height or leg length was observed [23].

In the pooled analysis of melanoma in women performed by Olsen et al [22], there was an increased risk of melanoma in the highest quartile of height compared with the lowest quartile for women overall (pooled odds ratio 1.3, 95\% CI 1.1-1.6). After stratification by age, the association was limited to women $<50$ years of age (pooled odds ratio 1.4, 95\% CI 1.1-1.9). Elevated odds ratios in the highest quartile of height were found for superficial spreading melanoma (pooled odds ratio $1.3,95 \%$ CI 1.0-1.7), and to a lesser extent for nodular melanoma (pooled odds ratio 1.2, 95\% CI 0.7-2.1), whereas no association with lentigo maligna melanoma was observed. Furthermore, increased risks associated with the highest quartile of height were 
noticed for melanomas of the head/neck, lower limbs, and upper limbs [22].

According to Thune et al [15], there was a positive association with height for melanomas localized on the face and trunk in both sexes, and for those of the lower limbs only in females. Stenehjem et al described significant positive trends for trunk, head, and neck melanomas in men, and for melanomas of the upper and lower limbs in women, and, as concerns the relation between height and histological subtype, they observed significant dose-related risks for superficial spreading melanoma and nodular melanoma in both sexes [25].

With regard to the studies shown in Table 1 , a great heterogeneity is recognizable among them, in terms of design, sample size and characteristics, as well as adjusted confounding factors. Limitations of most studies were the lack of complete information on several variables known to be risk factors for cutaneous melanoma and consequently the inability to adjust for the potential confounding effect of such variables. For instance, only some studies included adjustment for sunlight exposure, sun sensitivity (eg, sunburn) and/or UVR indicators, hair color and/or other pigmentary traits, and number of nevi (Table 1). Nevertheless, such information was categorized using nonstandardized and heterogeneous definitions, especially in the case of nevi count and sunlight exposure. Lahmann et al took into account in their model clinical elastosis of the neck as an indicator of sun sensitivity and cumulative sun exposure [24]. In very few studies, data on family history of melanoma, other skin cancers or malignancies, or personal history of nonmelanoma skin cancer were available and taken into consideration (Table 1).

In the analysis performed by Kvaskoff et al [23], the positive association between height and melanoma, detected in age-adjusted models only, was weakened and lost any statistical significance after full adjustment for age and also for other known risk factors for melanoma. In particular, the factors that decreased the strength of this association were number of nevi and hair color. The associations revealed by Olsen et al were instead unaffected by adjustment for other known melanoma risk factors, such as sun exposure-related features [22].

Many other factors may act as confounders, including ethnicity, socioeconomic status, education, outdoor activity, smoking, tanning habit, use of sunbeds, and other lifestyle factors, and these were taken into consideration occasionally (Table 1 ).

Moreover, the majority of studies have relied on selfreported height, thus impairing the accurateness and precision of data entry [1]. In fact, only a limited number of reports clearly indicated that measurement of height was obtained by trained staff $[15,21,24,25]$. Inaccuracy of estimates might also be related to recall bias, when the self-reporting of height was done in different periods of time, or to the assess- ment of height after the occurrence of cancer in case-control studies [22,25].

It is relevant to underline that a recent systematic review and meta-analysis gave evidence for the relationship between height and melanoma risk [26]. The authors included 12 prospective studies, most of which enrolled Caucasian populations with a total of 4,723,739 participants and 20,049 cases of melanoma. Different potential confounding factors were taken into account for multivariate regression analyses in the included studies. In brief, the cumulative findings of this meta-analysis indicated that subjects in the top category of height had increased risk of melanoma (random-effects relative risk 1.46, 95\% CI 1.24-1.73; P < 0.001) compared with the lowest category. Per $10-\mathrm{cm}$ increment in height was positively correlated with increased melanoma risk (randomeffects relative risk 1.27, 95\% CI 1.19-1.35; P < 0.001), with significant results seen in both women and men using a subgroup analysis by gender.

\section{Childhood Height}

Meyle et al examined anthropometric parameters in childhood (measured at ages 7-13 years) in relation to future risk of melanoma. For this purpose, they analyzed the data of 372,636 Danish children from the Copenhagen School Health Records Register, during approximately 11 million person-years of follow-up [27]. Relevant strengths of this study were the large sample size, the minimal loss to followup, the absence of selection bias, the inclusion of carefully recorded height measurements, and the identification of melanoma cases via linkage to the national cancer registry. Greater childhood height at the ages of 7 and 13 years was shown to be significantly associated with an increased risk of melanoma in adulthood. Melanoma risk was also associated with birth weight. Therefore, the authors concluded that associations between body size and melanoma may initiate early in life and can be related to height and birth weight, without any apparent influence of body mass index or body surface area [27].

A further analysis of the same cohort revealed the positive and significant association of childhood height with the majority of melanoma variants in adulthood (superficial spreading melanoma, nodular melanoma, melanoma not otherwise specified), but not with lentigo maligna melanoma [28].

\section{Putative Mechanisms Underlying Height-Melanoma Relationship}

In various analyses, positive height-cancer gradients were disclosed for many cancer types, including melanoma [3-10], suggesting that factors related to height might contribute 
to cancer development in adulthood. The observations that height can be associated with selected cancers but not others seem to imply the existence of biological and behavioral mechanisms that vary depending on the anatomical site but may be shared by different cancer sites and/or types $[3,8]$.

A number of hypotheses have been proposed to explain the association between height and melanoma.

Human growth is a complex phenomenon influenced by genetic, hormonal, nutritional, and other environmental factors from fetal life to puberty. The growth hormone (GH)-insulin-like growth factor (IGF)-1 axis plays a central role in growth. Nevertheless, numerous genes are involved in the control of stature, the large part of which are outside the GH-IGF-1 system, and many other molecules, as well as multiple intracellular molecular pathways, are involved in growth processes [29]. For instance, a meta-analysis of genome-wide association studies identified nearly 700 variants, reflecting 423 loci, that contribute to normal adult stature variation in individuals of European ancestry and can explain nearly 20\% of the heritability for adult height [30].

Adult attained height is thought to be the consequence of the interplay of genetic factors and many early-life experiences and exposures that may have an impact on cancer or other pathological events occurring much later in life. Stature may be an indirect indicator of nutritional status or caloric intake during childhood or adolescence [31]. A few data have suggested that caloric restriction during development can have lasting beneficial effect on the future risk of malignancy [32].

It has been postulated that cancer risk is proportional to organ cellularity and the number of proliferating cells within tissues [3,31]. Height is directly correlated with the number of cells that can be susceptible to convert into neoplastic cells, and, for melanoma, the number of melanocytes is relevant, as well as the total body area. Some authors detected a positive association between melanoma risk and high body surface area [15,25], while others noted no relation with body surface area [22,23]. As concerns nevus number, Ribero et al highlighted the importance of growth in melanoma susceptibility based on the results of a study in the TwinsUK cohort that disclosed the positive association between nevus count, an important risk factor for melanoma, and height [33]. Moreover, their study also revealed that nevus count was unrelated to weight and positively associated with bone mineral density. Adjustment for leukocyte telomere length, known to be linked to both bone mineral density and nevus count, did not modify the significance of the association between height and nevus count, thereby ruling out a direct influence of leukocyte telomere length on the results [33].

Height is correlated with hormones and growth factors that may also be involved in carcinogenesis. The IGF system plays an important role during the peripubertal growth spurt, and the peak in height velocity at adolescence is driven by a cascade of hormones, primarily initiated by a rise in sex steroids and followed by increases in GH and the related IGF-1 [34]. IGF-1 level in children was found to be positively associated with growth in height [35]. Moreover, the GH-IGF-1 axis can be influenced by nutritional status [36]. IGF and GH have been implicated not only in tissue growth and development, but also in carcinogenesis [37]. Results from prospective studies of individuals with acromegaly have reported increased risks of melanoma [38]. $\mathrm{GH}$ exerts multiple biological effects directly or through IGF-1 action and can interfere with many intracellular signaling pathways known to be implicated in oncogenesis [37]. Of interest, human melanoma cells have been found to express $\mathrm{GH}$ receptors and also human metastatic melanoma cells were shown to possess active $\mathrm{GH}$ receptors that can modulate multiple signaling pathways capable of promoting tumor progression [39-41]. In addition, experimental data have supported the regulatory role of IGF axis in human melanoma, particularly in the proliferation of early-stage melanoma cells [41-43].

Previous case-control studies have examined the association between circulating IGF-1 concentrations and the risk of melanoma with inconsistent results [44,45], while a nested case-control study in a prospective cohort from 10 European countries did not find any significant association between circulating IGF-1 concentration measured in adulthood and melanoma risk [46]. However, these findings do not necessarily rule out the influence of IGF-1, especially during childhood, on the future risk of melanoma. As previously mentioned, the achieved height can be correlated with the level of serum IGF-1 in puberty, but later any correlation may be lost. Growth-influencing exposures during childhood, which operate also through effects on IGF-1 levels, may have long-term influences on disease risk, without, however, conditioning IGF-1 levels throughout life [47]. In this context, prepubertal age is likely to be a crucial period in determining the risk of melanoma in adulthood, as suggested by the effects of sunburn and UVR exposure at these ages on nevogenesis and melanomagenesis [48].

A recent hypothesis has taken into consideration bodyresonant radiation that may affect both cancer incidence and body height. Radio-waves might influence body height through hormonal disturbances. It has been speculated that individuals who sleep in a bed that acts as a radio-wave antenna can be particularly prone to the harmful effects of radiation. Therefore, a correlation between melanoma incidence, mean body height, the number of FM broadcasting transmitters, as well as the use of metal spring mattresses has been hypothesized [49]. 


\section{Conclusions}

Accumulating evidence suggests the relation between anthropometry and cancer. A positive association between height and melanoma risk has been suggested, although results appear to be still controversial. However, the existence of a significant association has been highlighted in a recent systematic review and meta-analysis [26].

Further research is, however, required to confirm a heightmelanoma relationship and better understand the underlying mechanisms. An intriguing aspect is the identification of the possible shared genetic factors and pathomechanisms, as well as exposures occurring early in life that are capable of influencing both growth process and future cancer risk.

\section{References}

1. Bandera EV, Fay SH, Giovannucci E, et al; World Cancer Research Fund International Continuous Update Project Panel. The use and interpretation of anthropometric measures in cancer epidemiology: a perspective from the world cancer research fund international continuous update project. Int J Cancer. 2016;139(11):2391-2397.

2. Rastrelli M, Tropea S, Rossi CR, Alaibac M. Melanoma: epidemiology, risk factors, pathogenesis, diagnosis and classification. In Vivo. 2014;28(6):1005-1011.

3. Walter RB, Brasky TM, Buckley SA, et al. Height as an explanatory factor for sex differences in human cancer. J Natl Cancer Inst. 2013;105(12):860-868.

4. Wirén S, Häggström C, Ulmer H, et al. Pooled cohort study on height and risk of cancer and cancer death. Cancer Causes Control. 2014;25(2):151-159.

5. Kabat GC, Kim MY, Hollenbeck AR, Rohan TE. Attained height, sex, and risk of cancer at different anatomic sites in the NIH-AARP diet and health study. Cancer Causes Control. 2014;25(12):1697-1706.

6. Jiang Y, Marshall RJ, Walpole SC, et al. An international ecological study of adult height in relation to cancer incidence for 24 anatomical sites. Cancer Causes Control. 2015;26(3):493-499.

7. Green J, Cairns BJ, Casabonne D, et al; Million Women Study Collaborators. Height and cancer incidence in the Million Women Study: prospective cohort, and meta-analysis of prospective studies of height and total cancer risk. Lancet Oncol. 2011;12(8):785794.

8. Kabat GC, Anderson ML, Heo M, et al. Adult stature and risk of cancer at different anatomic sites in a cohort of postmenopausal women. Cancer Epidemiol Biomarkers Prev. 2013;22(8):13531363.

9. Kabat GC, Heo M, Kamensky V, et al. Adult height in relation to risk of cancer in a cohort of Canadian women. Int J Cancer. 2013;132(5):1125-1132.

10. Yang TO, Reeves GK, Green J, et al; Million Women Study Collaborators. Birth weight and adult cancer incidence: large prospective study and meta-analysis. Ann Oncol. 2014;25(9):1836-1843.

11. Emerging Risk Factors Collaboration. Adult height and the risk of cause-specific death and vascular morbidity in 1 million people: individual participant meta-analysis. Int J Epidemiol. 2012;41(5):1419-1433.
12. Batty GD, Shipley MJ, Langenberg C, et al. Adult height in relation to mortality from 14 cancer sites in men in London (UK): evidence from the original Whitehall study. Ann Oncol. 2006;17(1):157-166.

13. Veierød MB, Thelle DS, Laake P. Diet and risk of cutaneous malignant melanoma: a prospective study of 50,757 Norwegian men and women. Int J Cancer. 1997;71(4):600-604.

14. Cutler C, Foulkes WD, Brunet JS, et al. Cutaneous malignant melanoma in women is uncommonly associated with a family history of melanoma in first-degree relatives: a case-control study. Melanoma Res. 1996;6(6):435-440.

15. Thune I, Olsen A, Albrektsen G, Tretli S. Cutaneous malignant melanoma: association with height, weight and body-surface area. a prospective study in Norway. Int J Cancer. 1993;55(4):555-561.

16. Freedman DM, Sigurdson A, Doody MM, et al. Risk of melanoma in relation to smoking, alcohol intake, and other factors in a large occupational cohort. Cancer Causes Control. 2003;14(9):847857.

17. Shors AR, Solomon C, McTiernan A, White E. Melanoma risk in relation to height, weight, and exercise (United States). Cancer Causes Control. 2001;12(7):599-606.

18. Dubin N, Moseson M, Pasternack BS. Epidemiology of malignant melanoma: pigmentary traits, ultraviolet radiation, and the identification of high-risk populations. Recent Results Cancer Res. 1986;102:56-75.

19. Gallus S, Naldi L, Martin L, et al; Oncology Study Group of the Italian Group for Epidemiologic Research in Dermatology (GISED). Anthropometric measures and risk of cutaneous malignant melanoma: a case-control study from Italy. Melanoma Res. 2006;16(1):83-87.

20. Dennis LK, Lowe JB, Lynch CF, Alavanja MC. Cutaneous melanoma and obesity in the Agricultural Health Study. Ann Epidemiol. 2008;18(3):214-221.

21. Levine H, Afek A, Shamiss A, et al. Country of origin, age at migration and risk of cutaneous melanoma: a migrant cohort study of 1,100,000 Israeli men. Int J Cancer. 2013;133(2):486-494.

22. Olsen CM, Green AC, Zens MS, et al. Anthropometric factors and risk of melanoma in women: a pooled analysis. Int J Cancer. 2008;122(5):1100-1108.

23. Kvaskoff M, Bijon A, Mesrine S, et al. Anthropometric features and cutaneous melanoma risk: a prospective cohort study in French women. Cancer Epidemiol. 2014;38(4):357-363.

24. Lahmann PH, Hughes MC, Williams GM, Green AC. A prospective study of measured body size and height and risk of keratinocyte cancers and melanoma. Cancer Epidemiol. 2016;40:119-125.

25. Stenehjem JS, Veierød MB, Nilsen LT, et al. Anthropometric factors and cutaneous melanoma: prospective data from the population-based Janus Cohort. Int J Cancer. 2018;142(4):681690.

26. Yu D-J, Li X-J, Morice A, et al. Height and risk of melanoma: a systematic review and meta-analysis. Int J Clin Exp Med. 2018;11(5):4426-4435.

27. Meyle KD, Gamborg M, Sørensen TIA, Baker JL. Childhood body size and the risk of malignant melanoma in adulthood. Am J Epidemiol. 2017;185(8):673-680.

28. Meyle KD, Gamborg M, Hölmich LR, Baker JL. Associations between childhood height and morphologically different variants of melanoma in adulthood. Eur J Cancer. 2016;67:99-105.

29. Baron J, Sävendahl L, De Luca F, et al. Short and tall stature: a new paradigm emerges. Nat Rev Endocrinol. 2015;11(12):735-746. 
30. Wood AR, Esko T, Yang J, et al. Defining the role of common variation in the genomic and biological architecture of adult human height. Nat Genet. 2014;46(11):1173-1186.

31. Albanes D, Winick M. Are cell number and cell proliferation risk factors for cancer? J Natl Cancer Inst. 1988;80(10):772-774.

32. Frankel S, Gunnell DJ, Peters TJ, et al. Childhood energy intake and adult mortality from cancer: the Boyd Orr Cohort Study. BMJ. 1998;316(7130):499-504.

33. Ribero S, Glass D, Aviv A, et al. Height and bone mineral density are associated with naevus count supporting the importance of growth in melanoma susceptibility. PLoS One. 2015;10(1):e0116863.

34. Murray PG, Clayton PE. Endocrine control of growth. Am J Med Genet C Semin Med Genet. 2013;163C(2):76-85.

35. Rogers I, Metcalfe C, Gunnell D, et al. Avon Longitudinal Study of Parents Children Study Team. Insulin-like growth factor-I and growth in height, leg length, and trunk length between ages 5 and 10 years. J Clin Endocrinol Metab. 2006;91(7):2514-2519.

36. Hawkes CP, Grimberg A. Insulin-like growth factor-I is a marker for the nutritional state. Pediatr Endocrinol Rev. 2015;13(2):499511.

37. Chhabra Y, Waters MJ, Brooks AJ. Role of the growth hormone-IGF-1 axis in cancer. Expert Rev Endocrinol Metab. 2011;6(1):71-84.

38. Gunnell D, Okasha M, Smith GD, et al. Height, leg length, and cancer risk: a systematic review. Epidemiol Rev. 2001;23(2):313342 .

39. Lincoln DT, Sinowatz F, Kölle S, et al. Up-regulation of growth hormone receptor immunoreactivity in human melanoma. Anticancer Res. 1999;19(3A):1919-1931.

40. Sustarsic EG, Junnila RK, Kopchick JJ. Human metastatic melanoma cell lines express high levels of growth hormone receptor and respond to GH treatment. Biochem Biophys Res Commun. 2013;441(1):144-150.

41. Basu R, Wu S, Kopchick JJ. Targeting growth hormone receptor in human melanoma cells attenuates tumor progression and epithelial mesenchymal transition via suppression of multiple oncogenic pathways. Oncotarget. 2017;8(13):21579-21598.

42. Satyamoorthy K, Li G, Vaidya B, et al. Insulin-like growth factor-1 induces survival and growth of biologically early melanoma cells through both the mitogen-activated protein kinase and betacatenin pathways. Cancer Res. 2001;61(19):7318-7324.

43. Capoluongo E. Insulin-like growth factor system and sporadic malignant melanoma. Am J Pathol. 2011;178(1):26-31.

44. Park SL, Setiawan VW, Kanetsky PA, et al. Serum insulin-like growth factor-I and insulin-like growth factor binding protein-3 levels with risk of malignant melanoma. Cancer Causes Control. 2011;22(9):1267-1275.

45. Kucera R, Treskova I, Vrzalova J, et al. Evaluation of IGF1 serum levels in malignant melanoma and healthy subjects. Anticancer Res. 2014;34(9):5217-5220.

46. Bradbury KE, Appleby PN, Tipper SJ, et al. Circulating insulinlike growth factor I in relation to melanoma risk in the European Prospective Investigation into Cancer and Nutrition. Int J Cancer. 2019;144(5):957-966.

47. Gunnell D, Oliver SE, Donovan JL, et al. Do height-related variations in insulin-like growth factors underlie the associations of stature with adult chronic disease? J Clin Endocrinol Metab. 2004;89(1):213-218.

48. Green AC, Wallingford SC, McBride P. Childhood exposure to ultraviolet radiation and harmful skin effects: epidemiological evidence. Prog Biophys Mol Biol. 2011;107(3):349-355.

49. Hallberg O. Cancer and body height. Pathophysiology. 2014;21(2):177-181. 